Annals of Pure and Applied Mathematics

Vol. 17, No. 2, 2018, 157-162

ISSN: 2279-087X (P), 2279-0888(online)

Published on 28 May 2018

Annals of

www.researchmathsci.org

DOI: http://dx.doi.org/10.22457/apam.v17n2a2

Pure and Applied

Mathematics

\title{
A New Numerical Approach for Solving Initial Value Problems of Ordinary Differential Equations
}

\author{
Md. Kamruzzaman ${ }^{1}$ and Md. Mehedi Hasan ${ }^{2}$ \\ ${ }^{1}$ Department of Civil Engineering \\ Presidency University, Gulshan, Dhaka, Bangladesh \\ ${ }^{2}$ Daffodil International University, Dhanmondi, Dhaka, Bangladesh \\ Email: mehedi.ged@diu.edu.bd \\ ${ }^{1}$ Corresponding author. Email: zzamank@ pu.edu.bd
}

Received 1 April 2018; accepted 25 May 2018

\begin{abstract}
In this article, we developed a new numerical approach which is mainly concentrates to solve some complicated initial value problems of ordinary differential equations. The complete breakdown of this new approach derivation is presented here. In our future work, we will examine on the main properties of the technique namely consistency, convergence and feasibility. The expansion of this new numerical scheme shall be worked-on and comparison is also be made with some existing methods.
\end{abstract}

Keywords: Numerical approach, ordinary differential equations, numerical scheme expansion

\section{AMS Mathematics Subject Classification (2010): 93C15}

\section{Introduction}

In mathematical modeling differential equations are generally used in the field of science and engineering. In the field of mathematical physics different problems are arise as the form of differential equations. These types of differential equations may the formation either ordinary differential equations or partial differential equations. Practically, most of the models of the problem which are formulated by means of these equations are so complicated to determine the exact solution and one of two approaches is taken to approximate the solution. The first technique is to reduce the differential equation in to one that can be solved exactly and then use the result of the reduced equation to approximate the solution to the original problem. Another technique, which we will verify in this article, uses methods of approximations the solution of original problem. This is the technique that is generally taken as the approximation methods give more perfect results and relative error information. Numerical methods are usually used for solving mathematical problems that are articulated in the field of science and engineering 
Md. Kamruzzaman and Md. Mehedi Hasan

in which case the determination of the exact solution is so hard or impossible. Only a few numbers of differential equations can be solved analytically. Consequently, to obtain the analytical solution for differential equation there exist different methods. A huge number of differential equations are unable to determine the solution in closed form using familiar analytical methods, in which case we apply numerical technique for solving a differential equation under certain initial restriction or restrictions. There exist different kinds of practical numerical methods for finding the solution of initial value problem of ordinary differential equations.

Many Numerical researchers namely Ogunrinde [1], Fatunla [2], Butcher [3], Liu, Turner [4] and even Ibijola [5] and so on, have established schemes in order to solve some initial value problems of ordinary differential equations. Enright, Fellen and Sedgwick [6] have developed a numerical method which compares the numerical solution of ordinary differential equations. Yuan and Agrawal [7], established a scheme for fractional derivatives, on the other hand Obaymi [8,9] also studied on some approximation techniques which were used to derive stable non-standard finite difference schemes. And Ibijola [10] focus on the convergence, consistency and stability of a method of integration of ordinary differential equations. [11-13] solved ODE with numerical examples. The efficiency of all their efforts made for stability, accuracy, convergence and consistency of the methods. The accuracy property of different methods can be considered an order and convergence as well as truncation error co-efficient.

In this paper, we have established a new numerical technique with some particular properties to determine the solution of initial value problems of ordinary differential equations based on the local representation of theoretical form.

Let us consider

$y^{\prime}=\frac{d y}{d x}=f(x, y), \quad y(a)=y_{0}$

is interpolating by the function

$F(x)=C_{0}+C_{1} x^{2}+C_{2} e^{x^{2}-1}+D \sin \left(x^{2}-1\right)$

where $C_{0}, C_{1}, C_{2}$ and $D$ are real undetermined co-efficients.

\section{Derivation of the new approach}

Let us suppose that $y_{k}$ is a numerical estimation of the theoretical solution $y(x)$ and $f_{k}=f\left(x_{k}, y_{k}\right)$.

Now we define the mesh points as follows: $x_{k}=a+k h ; k=0,1,2,3, \ldots \ldots \ldots$.

We proceed to derive the new technique is as follows:

$F^{\prime}(x)=2 C_{1} x+2 C_{2} x e^{x^{2}-1}+2 D x \cos \left(x^{2}-1\right)$

$F^{\prime \prime}(x)=2 C_{1}+2 C_{2}\left(2 x^{2} e^{x^{2}-1}+e^{x^{2}-1}\right)-2 D\left\{2 x^{2} \sin \left(x^{2}-1\right)-\cos \left(x^{2}-1\right)\right\}$

Similarly,

$F^{\prime \prime \prime}(x)=2 C_{2}\left(4 x^{3} e^{x^{2}-1}+6 x e^{x^{2}-1}\right)-2 D\left\{4 x^{3} \cos \left(x^{2}-1\right)+6 x \sin \left(x^{2}-1\right)\right\}$ 
A New Numerical Approach for Solving Initial Value Problems of Ordinary Differential Equations

$F^{i v}(x)=2 C_{2}\left(8 x^{4} e^{x^{2}-1}+24 x^{2} e^{x^{2}-1}+6 e^{x^{2}-1}\right)-2 D\left\{-8 x^{4} \sin \left(x^{2}-1\right)+\right.$ $\left.24 x^{2} \cos \left(x^{2}-1\right)+6 \sin \left(x^{2}-1\right)\right\}$

From equation (2), we have,

$$
F(x)=C_{0}+C_{1} x+C_{2} e^{x^{2}-1}+D \sin \left(x^{2}-1\right)
$$

Or, $C_{0}=F(x)-C_{1} x-C_{2} e^{x^{2}-1}-D \sin \left(x^{2}-1\right)$

And from equation (3), we get,

$C_{1}=\frac{F^{\prime}(x)}{2 x}-C_{2} e^{x^{2}-1}-D \cos \left(x^{2}-1\right)$

From equation (4), we get,

$C_{2}=\frac{F^{\prime \prime}(x)-2 C_{1}+2 D\left\{2 x^{2} \sin \left(x^{2}-1\right)-\cos \left(x^{2}-1\right)\right\}}{2\left(2 x^{2} e^{x^{2}-1}+e^{x^{2}-1}\right)}$

Also from equation (5), we get,

$D=\frac{F^{\prime \prime \prime}(x)-2 C_{2}\left(4 x^{3} e^{x^{2}-1}+6 x e^{x^{2}-1}\right)}{-2\left\{4 x^{3} \cos \left(x^{2}-1\right)+6 x \sin \left(x^{2}-1\right)\right\}}$

Now substituting the value of $C_{1}$ from (8) into (9), we get,

$C_{2}=\frac{F^{\prime \prime}(x)-F^{\prime}(x)+2 D\left\{x \cos \left(x^{2}-1\right)-\cos \left(x^{2}-1\right)\right\}+4 D x^{2} \sin \left(x^{2}-1\right)}{4 x^{3} e^{x^{2}-1}}$

Putting the value of $C_{2}$ from (11) into (10), we get,

$$
D=\frac{x^{4} F^{\prime \prime \prime}(x)+\left(-2 x^{4}-3 x^{2}\right) F^{\prime \prime}(x)+\left(2 x^{4}+3 x^{2}\right) F^{\prime}(x)}{\left\{-8 \mathrm{x}^{7}+2 \mathrm{x}^{5}-2 \mathrm{x}^{4}\right\} \cos \left(x^{2}-1\right)+\left\{-12 x^{5}+8 x^{8}+3\right\} \sin \left(x^{2}-1\right)}
$$

By substituting the value of D from (12) into (11), we get,

$$
C_{2}=\frac{\begin{array}{c}
\left.\left[6 x^{2}-6 x^{3}-2 x^{4}+2 x^{5}+8 \mathrm{x}^{7}\right) \cos \left(x^{2}-1\right)+\left(-12 x^{4}+12 x^{5}+8 x^{6}-8 x^{8}-3\right) \sin \left(x^{2}-1\right)\right] F^{\prime}(x) \\
+\left[\left\{6 x^{3}-4 x^{4}-2 x^{5}-8 \mathrm{x}^{7}\right\} \cos \left(x^{2}-1\right)+\left\{-24 x^{5}-8 x^{6}+8 x^{8}+3\right\} \sin \left(x^{2}-1\right)\right] F^{\prime \prime}(x) \\
+\left\{\left(2 x^{5}-2 x^{4}\right) \cos \left(x^{2}-1\right)+4 x^{6} \sin \left(x^{2}-1\right)\right\} F^{\prime \prime \prime}(x)
\end{array}}{\left[\left\{-8 \mathrm{x}^{7}+2 \mathrm{x}^{5}-2 \mathrm{x}^{4}\right\} \cos \left(x^{2}-1\right)+\left\{-12 x^{5}+8 x^{8}+3\right\} \sin \left(x^{2}-1\right)\right] 4 x^{3} e^{x^{2}-1}}
$$

Putting the value of $\mathrm{D}$ from (11) and $C_{2}$ from (12) into (8), we get,

$$
\begin{gathered}
C_{1}=\frac{1}{2 x} F^{\prime}(x)-C_{2} e^{x^{2}-1}-D \cos \left(x^{2}-1\right)=\frac{1}{2 x} F^{\prime}(x) \\
-\left\{\frac{\begin{array}{c}
{\left[\left(6 x^{2}-6 x^{3}-2 x^{4}+2 x^{5}+8 x^{7}\right) \cos \left(x^{2}-1\right)+\left(-12 x^{4}+12 x^{5}+8 x^{6}-8 x^{8}-3\right) \sin \left(x^{2}-1\right)\right] F^{\prime}(x)} \\
+\left[\left\{6 x^{3}-4 x^{4}-2 x^{5}-8 x^{7}\right\} \cos \left(x^{2}-1\right)+\left\{-24 x^{5}-8 x^{6}+8 x^{8}+3\right\} \sin \left(x^{2}-1\right)\right] F^{\prime \prime}(x) \\
+\left\{\left(2 x^{5}-2 x^{4}\right) \cos \left(x^{2}-1\right)+4 x^{6} \sin \left(x^{2}-1\right)\right\} F^{\prime \prime \prime}(x)
\end{array}}{\left[\left\{-8 \mathrm{x}^{7}+2 \mathrm{x}^{5}-2 \mathrm{x}^{4}\right\} \cos \left(x^{2}-1\right)+\left\{-12 x^{5}+8 x^{8}+3\right\} \sin \left(x^{2}-1\right)\right] 4 x^{3}}\right. \\
-\cos \left(x^{2}-1\right)\left\{\frac{x^{4} F^{\prime \prime \prime}(x)+\left(-2 x^{4}-3 x^{2}\right) F^{\prime \prime}(x)+\left(2 x^{4}+3 x^{2}\right) F^{\prime}(x)}{\left\{-8 \mathrm{x}^{7}+2 \mathrm{x}^{5}-2 \mathrm{x}^{4}\right\} \cos \left(x^{2}-1\right)+\left\{-12 x^{5}+8 x^{8}+3\right\} \sin \left(x^{2}-1\right)}\right\}
\end{gathered}
$$

Let us consider,

$$
P=\left\{\frac{\begin{array}{c}
{\left[\left(6 x^{2}-6 x^{3}-2 x^{4}+2 x^{5}+8 \mathrm{x}^{7}\right) \cos \left(x^{2}-1\right)+\left(-12 x^{4}+12 x^{5}+8 x^{6}-8 x^{8}-3\right) \sin \left(x^{2}-1\right)\right] F^{\prime}(x)} \\
+\left[\left\{6 x^{3}-4 x^{4}-2 x^{5}-8 \mathrm{x}^{7}\right\} \cos \left(x^{2}-1\right)+\left\{-24 x^{5}-8 x^{6}+8 x^{8}+3\right\} \sin \left(x^{2}-1\right)\right] F^{\prime \prime}(x) \\
+\left\{\left(2 x^{5}-2 x^{4}\right) \cos \left(x^{2}-1\right)+4 x^{6} \sin \left(x^{2}-1\right)\right\} F^{\prime \prime \prime}(x)
\end{array}}{\left[\left\{-8 \mathrm{x}^{7}+2 \mathrm{x}^{5}-2 \mathrm{x}^{4}\right\} \cos \left(x^{2}-1\right)+\left\{-12 x^{5}+8 x^{8}+3\right\} \sin \left(x^{2}-1\right)\right] 4 x^{3}}\right\}
$$


Md. Kamruzzaman and Md. Mehedi Hasan

And $Q=\cos \left(x^{2}-1\right)\left\{\frac{x^{4} F^{\prime \prime \prime}(x)+\left(-2 x^{4}-3 x^{2}\right) F^{\prime \prime}(x)+\left(2 x^{4}+3 x^{2}\right) F^{\prime}(x)}{\left\{-8 \mathrm{x}^{7}+2 \mathrm{x}^{5}-2 \mathrm{x}^{4}\right\} \cos \left(x^{2}-1\right)+\left\{-12 x^{5}+8 x^{8}+3\right\} \sin \left(x^{2}-1\right)}\right\}$

Hence the equation (14) becomes,

$C_{1}=\frac{1}{2 x} F^{\prime}(x)-P-Q$

Now applying the following restrictions on the interpolating function (2) in the following way:

Condition 1. The interpolating function (2) must be coincide with the theoretical solution at $x=x_{k}$ and $x=x_{k+1}$, such that

$$
F\left(x_{k}\right)=C_{0}+C_{1} x_{k}^{2}+C_{2} e^{x_{k}^{2}-1}+D \sin \left(x_{k}^{2}-1\right)
$$

And $F\left(x_{k+1}\right)=C_{0}+C_{1} x_{k+1}^{2}+C_{2} e^{x_{k+1}^{2}-1}+D \sin \left(x_{k+1}^{2}-1\right)$

Condition 2. The derivatives $F^{\prime}(x), F^{\prime \prime}(x), F^{\prime \prime \prime}(x)$ and $F^{k}(x)$ are coincide with $f(x), f^{\prime}(x), f^{\prime \prime}(x)$ and $f^{k-1}(x)$ respectively, that is,

$$
\begin{gathered}
F^{\prime}(x)=f_{k} \\
F^{\prime \prime}(x)=f_{k}^{\prime} \\
F^{\prime \prime \prime}(x)=f_{k}^{\prime \prime} \\
F^{i v}(x)=f_{k}^{\prime \prime \prime}
\end{gathered}
$$

From the above conditions (1) and (2), it follows that, if $F\left(x_{k+1}\right)-F\left(x_{k}\right)=y_{k+1}-y_{k}$ Then we have,

$$
\begin{gathered}
C_{0}+C_{1} x_{k+1}^{2}+C_{2} e^{x_{k+1}^{2}-1}+D \sin \left(x_{k+1}^{2}-1\right)-\left\{C_{0}+C_{1} x_{k}^{2}+C_{2} e^{x_{k}^{2}-1}+D \sin \left(x_{k}^{2}\right.\right. \\
-1)\}=y_{k+1}-y_{k}
\end{gathered}
$$

so, $\quad y_{k+1}=y_{k}+C_{1}\left(x_{k+1}^{2}-x_{k}^{2}\right)+C_{2}\left(e^{x_{k+1}^{2}-1}-e^{x_{k}^{2}-1}\right)+D\left\{\sin \left(x_{k+1}^{2}-1\right)-\right.$ $\left.\sin \left(x_{k}^{2}-1\right)\right\}$

Let us assume that

$x_{k}=a+k h$ then $x_{k}^{2}=(a+k h)^{2}=a^{2}+2 a k h+(k h)^{2}$

Also $x_{k+1}=a+(k+1) h$ so $x_{k+1}^{2}=\{a+(k+1) h\}^{2}$

hence, $x_{k+1}^{2}=a^{2}+2 a k h+2 a h+(k h)^{2}+2 k h^{2}+h^{2}$

Now we calculate, $x_{k+1}^{2}-x_{k}^{2}=2 h(a+k h)+h^{2}$

Similarly,

$$
\begin{aligned}
& e^{x_{k+1}^{2}-1}-e^{x_{k}^{2}-1}=e^{a^{2}+2 a k h+2 a h+(k h)^{2}+2 k h^{2}+h^{2}-1}-e^{a^{2}+2 a k h+(k h)^{2}-1} \\
& e^{x_{k+1}^{2}-1}-e^{x_{k}^{2}-1}=e^{a^{2}+2 a k h+(k h)^{2}-1}\left[e^{2 h(a+k h)+h^{2}}-1\right]
\end{aligned}
$$

And

$\sin \left(x_{k+1}^{2}-1\right)-\sin \left(x_{k}^{2}-1\right)=\sin \left\{a^{2}+2 a k h+2 a h+(k h)^{2}+2 k h^{2}+h^{2}-1\right\}-$ $\sin \left\{a^{2}+2 a k h+(k h)^{2}-1\right\}$

Putting (17) through (21) into equation (16) then we obtain our required numerical approach 
A New Numerical Approach for Solving Initial Value Problems of Ordinary Differential Equations

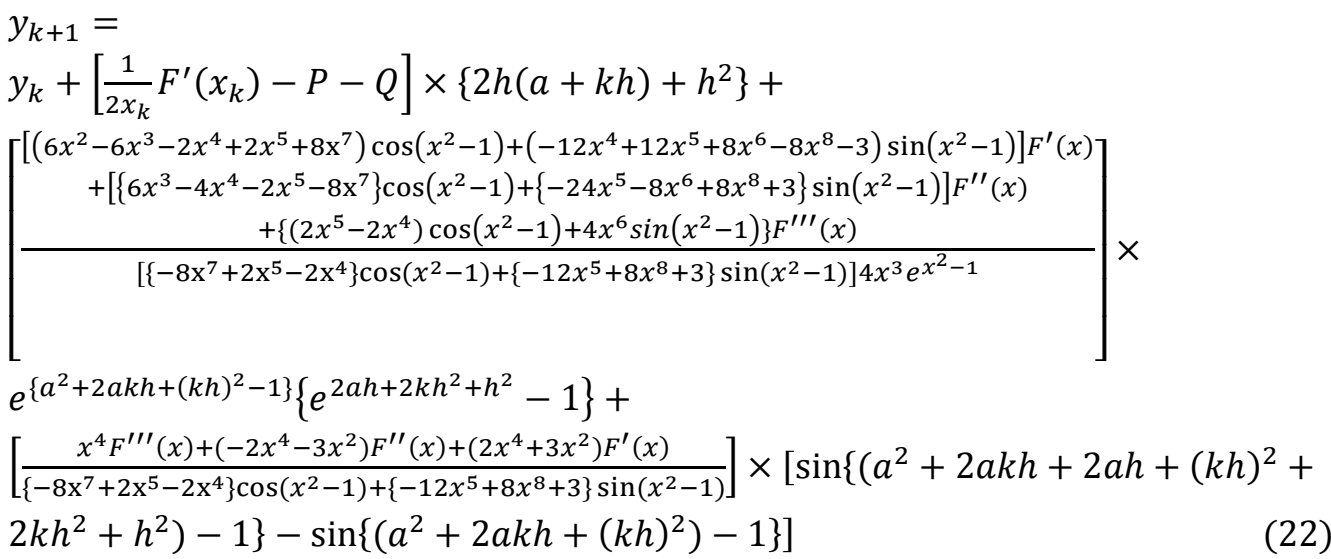

Equation (22) is the New Numerical Approach for the solution of ordinary differential equations with the given initial values.

\section{Conclusion}

Our main objective is to establish a new approach as a recommendation whose numerical approximation result could be coincides with some existing method of solution of different initial value problems of ordinary differential equations. So this paper has been capable to initiate the new approach as a proposal. In our future research, we will pay more concentration to validate this approach with some numerical examples. We will check some basic characteristics such as the accuracy, consistency, reliability, stability of the proposed new numerical approach by solving (1) and then discuss the relative error, truncation error on the comparison with some existing standard methods.

Acknowledgement: The authors thank to the anonymous referees for their valuable suggestions and comments which improved the presentation of the work.

\section{REFERENCES}

1. R.B.Ogunrinde, A new numerical scheme for the solution of initial value problem (IVP) in ordinary differential equations, Ph.D. Thesis, Ekiti State University, Ado Ekiti. (2010).

2. S.O.Fatunla, Numerical methods for initial value problems in ordinary differential equations, Academic Press Inc.(London), 308 (1988).

3. J.C.Butcher, Numerical solution for ordinary differential equation in the 20th century, Journal of Computational and Applied Mathematics, 125 (1-2) (2000) 1-29.

4. F.Liu, V.Anh and I.Turner, Numerical solution of space fractional Fokker-Planck equation, Journal of Computational and Applied Mathematics, 166(1) (2004) 209219.

5. E.A.Ibijola, A New Numerical Scheme for the Solution of Initial Value Problem, PhD Thesis, University of Benin, Nigeria, (1997). 
Md. Kamruzzaman and Md. Mehedi Hasan

6. T.E.Hull, W.H.Enright, B.M.Fellen and A.E.Sedgwick, Comparing numerical methods for ordinary differential equation, SLAM J. Numer. Anal, 9 (1972) 603-637.

7. L.Yuan and O.P.Agrawal, A numerical scheme for dynamic systems containing fractional derivatives, Vibration Acoustics, 124 (2002) 321-324.

8. A.A.Obayomi, A set of non-standard finite difference schemes for the solution of an equation of the type $y^{\prime}=y\left(1-y^{n}\right)$, International Journal of Pure and Applied Sciences and Technology, 12 (2012) 34-42.

9. A.A.Obayomi, Derivation of non-standard finite difference schemes for the second order chemical reaction model, Canadian Journal on Commuting in Mathematics, Natural Sciences, Engineering and Medicine, 3 (2012) 121-124.

10. E.A.Ibijola, On the convergence consistency and stability of a one step method for integration of ordinary differential equation, International Journal of Computer Mathematics, 73 (1998) 261-277.

11. P.Kama and E.A.Ibijola, On a new one - step method for numerical integration of ordinary differential equations, International Journal of Computer Mathematics, 78 (2000) 21-29.

12. J.D.Lambert, Numerical Methods for Ordinary Differential Systems: the Initial Value Problem, John Wiley \& Sons, Inc., New York, (1991).

13. Shepley L.Ross, Differential Equations. Third Edition, John Wiley \& Sons, Inc., New York (1984). 